\title{
A theorem of Semmes and the boundary absolute continuity in all dimensions
}

\section{Juha Heinonen}

Abstract. We use a recent theorem of Semmes to resolve some questions about the boundary absolute continuity of quasiconformal maps in space.

In [S3], Semmes proves that the quasisymmetric image of any set in $\mathbb{R}^{d}, d \geq 2$, of $d$-measure zero is again of $d$-measure zero. More formally, if $F \subset \mathbb{R}^{d}, d \geq 2$, and if $h: F \rightarrow \mathbb{R}^{d}$ is a quasisymmetric embedding, then

$$
\mathcal{H}_{d}(F)=0 \quad \text { if and only if } \quad \mathcal{H}_{d}(h(F))=0 .
$$

Here and hereafter, $\mathcal{H}_{p}$ denotes the $p$-dimensional Hausdorff measure for some positive integer $p$. Recall that an embedding $h: F \rightarrow \mathbb{R}^{d}$ is quasisymmetric if there is a homeomorphism $\eta:[0, \infty) \rightarrow[0, \infty)$ so that

$$
|x-a| \leq t|x-b| \quad \text { implies } \quad|h(x)-h(a)| \leq \eta(t)|h(x)-h(b)|,
$$

for all $x, a, b \in F$. Semmes's theorem has important consequences for the problem of boundary absolute continuity of quasiconformal maps in space, as will be explained in this note. In particular, we shall give a positive answer to Problem 1 in [H3, Section 6], and thereby improve upon the main result in [H2]. 
First we require some definitions. Suppose that $A \subset X \subset \mathbb{R}^{n}$. We say that $A$ is linearly locally connected in $X$ if there is a constant $C \geq 1$ so that, for all $a \in A$ and $r>0$,

1) points in $A \cap B(a, r)$ can be joined in $X \cap B(a, C r)$, and

2) points in $A \backslash \bar{B}(a, r)$ can be joined in $X \backslash \bar{B}(a, r / C)$.

Here joining means joining by a continuum, $B(z, t)$ is an open $n$-ball with center $z$ and radius $t$, and bar denotes the closure. The importance of the concept of linear local connectivity in the quasiconformal mapping theory was observed by Gehring in the sixties (in the nonrelative form, where $A=X$ ).

A metric space $Y$ is said to be a bi-Lipschitz $p$-ball if there is a bi-Lipschitz homeomorphism $\varphi$ of the open unit ball $\mathbb{B}^{p}$ of $\mathbb{R}^{p}$ onto $Y$. A metric space $E$ is said to be contained in a bi-Lipschitz $p$-ball $Y$ if there is an isometric embedding $i: E \rightarrow Y$.

Finally, recall (from geometric measure theory) that a subset of $\mathbb{R}^{n}$ is $p$-rectifiable if it is contained in a countable union of Lipschitz images of $\mathbb{R}^{p}$ plus a set of $\mathcal{H}_{p}$-measure zero; a set is purely $p$-unrectifiable if it contains no $p$-rectifiable subset of positive and finite $\mathcal{H}_{p}$-measure.

Theorem 1. Suppose that $f$ is a quasiconformal mapping of $\mathbb{B}^{n}$ onto a domain $D \subset \mathbb{R}^{n}, n \geq 3$, and that $A \subset D$ is bounded, pathwise connected, and linearly locally connected in $D$. If $E \subset \bar{A} \cap \partial D$ is such that $\mathcal{H}_{n-1}(E)=0$ and $\mathcal{H}_{n-1}\left(f^{-1}(E)\right)>0$, then there is no bi-Lipschitz $(n-1)$-ball containing $E$. If $E \subset \bar{A} \cap \partial D$ is such that $\mathcal{H}_{n-1}\left(f^{-1}(E)\right)=$ 0 , then $E$ is purely $(n-1)$-unrectifiable.

The meaning of $f^{-1}(E)$ will be explained in the proof below; note that a priori $f^{-1}$ is not defined on $\bar{A} \cap \partial D$. Also note that the second assertion is non-vacuous only if $E$ has positive, possibly infinite $(n-1)$ measure.

Theorem 1 says, in particular, that if $n \geq 3$, then a quasiconformal homeomorphism $f: \mathbb{B}^{n} \rightarrow D$ preserves the null sets of Hausdorff $(n-1)$ measure on the part of the boundary $\partial D$ that both lies on a bi-Lipschitz $(n-1)$-ball and can be touched from inside of $D$ by a nice subset $A$. Notice, however, that we do not require that $A$ meet the boundary in rectilinear cones, or anything like it; in principle, $A$ can be a fractal object wildly twisting and spiraling when approaching $\partial D$.

The first assertion in Theorem 1 is reminiscent of the Bishop-Jones theorem [BJ], which claims that if $f$ is a conformal map of $\mathbb{B}^{2}$ into the complex plane that maps (via its radial extension) a subset $E \subset \partial \mathbb{B}^{2}$ 
of positive length onto a set $f(E)$ of zero length, then $f(E)$ cannot lie on a rectifiable curve. It is well-known that Theorem 1 is false for quasiconformal maps in dimension $n=2$.

ProOF. The proof is simply a combination of Semmes's aforementioned theorem [S3] and the generalized subinvariance principle [H1, Theorem 6.6]. It follows from $[\mathrm{H} 1,6.6]$ that

$$
f^{-1}: A \longrightarrow f^{-1}(A)
$$

is a quasisymmetric map, and hence extends to a quasisymmetric map

$$
f^{-1}: \bar{A} \longrightarrow \overline{f^{-1}(A)} .
$$

We understand $f^{-1}(E) \subset \overline{f^{-1}(A)}$ precisely as the image of a set $E \subset$ $\bar{A} \cap \partial D$ under this extension, which is uniquely determined by $f$. Note that the inverse of a quasisymmetric map is quasisymmetric as well, so we have a quasisymmetric map

$$
\left(f^{-1}\right)^{-1}: \overline{f^{-1}(A)} \longrightarrow \bar{A}
$$

A couple of remarks need to be made here. The domains $D$ and $D^{\prime}$ in $[\mathrm{H} 1,6.6]$ are assumed to be bounded, but this is a redundant assumption which was unfortunately made in [H1]; if we only assume that $A$ is bounded, the same proof works verbatim. Also, the assumptions on $A$ in $[\mathrm{H} 1,6.6]$ are slightly differently phrased, but easily seen to be equivalent to the assumptions of Theorem 1 above.

Suppose now that $E \subset \bar{A} \cap \partial D$ satisfies $\mathcal{H}_{n-1}(E)=0$. Suppose also that $\varphi: \mathbb{B}^{n-1} \rightarrow Y$ is a bi-Lipschitz homeomorphism such that $Y$ contains $E$, and let $i: E \rightarrow Y$ be an isometric embedding. Then the quasisymmetric embedding

$$
h=\varphi^{-1} \circ i \circ\left(f^{-1}\right)^{-1}: f^{-1}(E) \longrightarrow \mathbb{R}^{n-1}
$$

maps the set $f^{-1}(E) \subset \partial \mathbb{B}^{n}$ into $\mathbb{R}^{n-1}$. Here $\left(f^{-1}\right)^{-1}$ is the map given in (2). Because $n-1 \geq 2$, we can apply Semmes's result to the map $h$. (The fact that the set $f^{-1}(E)$ lies on a smooth $(n-1)$-dimensional surface $\partial \mathbb{B}^{n}$ instead of $\mathbb{R}^{n-1}$ makes of course no difference here.) The conclusion $\mathcal{H}_{n-1}\left(f^{-1}(E)\right)=0$ then follows upon observing that biLipschitz maps preserve the null sets of every Hausdorff measure $\mathcal{H}_{p}$. This proves the first assertion of the theorem. 
The proof of the second assertion follows similarly. We need a theorem about Lipschitz maps (see $[\mathrm{F}, 3.2 .2]$ ): if $h: \mathbb{B}^{p} \rightarrow \mathbb{R}^{n}, 2 \leq$ $p \leq n$, is Lipschitz such that the image $h\left(\mathbb{B}^{p}\right)$ has positive $p$-measure, then there is a subset in $h\left(\mathbb{B}^{p}\right)$ of positive $p$-measure on which $h$ has a Lipschitz inverse. The rest is definition and Semmes's theorem. This completes the proof of Theorem 1 .

A point $w$ lying on the boundary of a domain $D$ in $\mathbb{R}^{n}$ is said to be an interior cone point of $D$ if, for some ball $B \subset D$, the cone

$$
w B=\{\lambda w+\mu x: \quad x \in B, \quad \lambda, \mu \geq 0, \text { with } \lambda+\mu=1\}
$$

lies in $D \cup\{w\}$. Note that the height and the opening of the cone is allowed to depend on $w$. Denote by $\mathcal{I C}_{D}$ the subset of $\partial D$ consisting of all the interior cone points of $D$.

Theorem 3. Suppose that $f$ is a quasiconformal mapping of $\mathbb{B}^{n}$ onto a domain $D \subset \mathbb{R}^{n}, n \geq 3$. Then, for any set $E \subset \mathcal{I C}_{D}$, we have that $\mathcal{H}_{n-1}(E)=0$ if and only if $\mathcal{H}_{n-1}\left(\dot{f}^{-1}(E)\right)=0$, where $\dot{f}$ denotes the radial extension of $f$ which exists outside a set of $n$-capacity zero, hence of Hausdorff dimension zero, on $\partial \mathbb{B}^{n}$.

REMARKS 4. a) The fact that $\dot{f}(z)=\lim _{r \rightarrow 1} f(r z)$ exists for $z \in \partial \mathbb{B}^{n}$ outside an exceptional set of zero $n$-capacity is well known. Moreover, an easy application of the quasiconformal Lindelöf's theorem shows that, for each $w \in \mathcal{I C}_{D}$, there is a point $z \in \partial \mathbb{B}^{n}$ such that $\dot{f}(z)=w$.

b) A weaker version of Theorem 3 where $\mathcal{I C}_{D}$ is replaced by the set of boundary points admitting both an exterior and interior cone, was proved in [H2] in dimensions $n \geq 3, n \neq 4$. Again, the theorem is false in dimension $n=2$.

Proof. Suppose that $E \subset \mathcal{I C}_{D}$ with $\mathcal{H}_{n-1}(E)>0$. It follows by standard arguments (cf. [H2, Proof of Theorem 4.3]) that there is a subset in $E$ of positive $\mathcal{H}_{n-1}$ measure that lies on the boundary of a biLipschitz $n$-ball $A$ contained in $D$. (The set $A$ is a union of cones of the form $w B, w \in E$, where $B$ has rational radius and rational coordinates for its center.) Because $A$ is a bi-Lipschitz ball, it is linearly locally connected and its boundary is a union of two bi-Lipschitz $(n-1)$-balls. It thus follows from Theorem 1 that $\mathcal{H}_{n-1}\left(\dot{f}^{-1}(E)\right)>0$. A similar argument shows that $\mathcal{H}_{n-1}\left(\dot{f}^{-1}(E)\right)=0$ if $\mathcal{H}_{n-1}(E)=0$ for $E \subset \mathcal{I C}_{D}$. The theorem follows. 
Theorem 1 is interesting, and new, already in the case when $A=D$, which is equivalent to $f$ being quasisymmetric in all of $\mathbb{B}^{n}$. A sufficient (but not necessary) condition for this occurrence is that $f$ be quasiconformally flat, i.e. $f$ extends to a quasiconformal homeomorphism $\mathbb{R}^{n} \rightarrow \mathbb{R}^{n}$. What is more, Semmes's result brings some new light into the absolute continuity properties of quasisymmetric embeddings of lower dimensional sets into $\mathbb{R}^{n}$. For completeness, we record the following theorem.

Theorem 5. Suppose that $E \subset \mathbb{R}^{p}$ and that $f: E \rightarrow \mathbb{R}^{n}$ is a quasisymmetric embedding, where $2 \leq p<n$. If $\mathcal{H}_{p}(E)>0$ and $\mathcal{H}_{p}(f(E))=0$, then there is no bi-Lipschitz p-ball containing $f(E)$. If $\mathcal{H}_{p}(E)=0$, then $f(E)$ is purely p-unrectifiable.

Proof. The first assertion is an immediate corollary of Semmes's theorem; the second assertion likewise reduces to it upon invoking $[\mathrm{F}$, 3.2.2] as in the proof of Theorem 1.

REMARKS 6. a) Gehring [G] (quasiconformally flat case) and Väisälä $[\mathrm{V}]$ (the general case) proved that if $f$ is a quasisymmetric embedding of an open set $G \subset \mathbb{R}^{p}$ into $\mathbb{R}^{n}$, where $2 \leq p<n$, and if the $p$-measure of $f(G)$ is finite, then $f$ is absolutely continuous; that is, $\mathcal{H}_{p}(f(E))=0$ if $\mathcal{H}_{p}(E)=0$ for $E \subset G$. It is not known, even if $f$ is quasiconformally flat, whether $f^{-1}$ is absolutely continuous in this case.

b) Stephen Semmes raised the interesting question whether it is always the case that the quasisymmetric image inside $\mathbb{R}^{n}$ of a set $E \subset$ $\mathbb{R}^{p}$ of positive $p$-measure has positive $p$-measure. Here $2 \leq p<n$. No counterexamples are known to me, and the only positive results that are known assume that the map is defined in a neighborhood of $E$ whose image is an Ahlfors-David p-regular set, $c f$. [S1, 3.4], [H2, 2.7]. In contrast to the equidimensional case required in [S3], the case $p<n$ is not symmetric any more in that a set $E \subset \mathbb{R}^{p}$ of zero $p$-measure may easily transform to a set of positive $p$-measure, or to a set of lower dimension, under a quasisymmetric embedding $f: E \rightarrow \mathbb{R}^{n}$; in fact, it is well known that this can happen for a global quasiconformal self map $f$ of $\mathbb{R}^{n}$. The point in Semmes's question is that $E$ has positive measure in top dimension. One may also wonder what happens if the range space $\mathbb{R}^{n}$ is replaced by an arbitrary metric space.

c) A question similar to the one in b) arises in the study of the boundary behavior of a quasiconformal map $f: \mathbb{B}^{n} \rightarrow \mathbb{R}^{n}$. Thinking of 
$f$ being defined capacity almost everywhere on $\partial \mathbb{B}^{n}$ ( $c f$. Remark 4.a)), one may ask how small can the set $f(E)$ be for $E \subset \partial \mathbb{B}^{n}$ of positive $(n-1)$-measure. To this effect, it was shown in [HK] that the Hausdorff dimension of $f(E)$ is at least $(2 K)^{1 /(1-n)}$, if $f$ is $K$-quasiconformal. It is possible that, for $n \geq 3$, the Hausdorff dimension of $f(E)$ has a lower bound that does not depend on $K$, and it is possible that this bound be $n-1$. If the latter is true, we would have an analog of Makarov's theorem for quasiconformal maps in space.

One is tempted to approach this problem by trying to embed $f(E)$ into $\mathbb{R}^{n-1}$ by a bi-Lipschitz map, if the dimension of $f(E)$ is low. Then Semmes's result would give a contradiction. However, such attempts are futile, as there are countable sets in $\mathbb{R}^{3}$ that do not admit bi-Lipschitz embeddings into $\mathbb{R}^{2}$. For example, one can take the three-fold Cartesian product of the sequence $\{1,1 / 2,1 / 3, \ldots\}$. (I learned this example from Jouni Luukkainen.)

d) One could replace throughout the text a bi-Lipschitz $p$-ball $Y$ by a quasisymmetric p-ball $Y$ that has the additional property that all quasisymmetric homeomorphisms $h: \mathbb{B}^{p} \rightarrow Y$ preserve null sets of Hausdorff $p$-measure. The family of such spaces $Y$ is known to be strictly larger than the family of bi-Lipschitz balls (see [S1], [S2]), but whether or not a given space is in this family is quite difficult to check. This is of course true for bi-Lipschitz balls as well; I simply chose to formulate the theorems in this paper in terms of the latter.

Acknowledgements. I thank Stephen Semmes and the referee for some useful remarks.

\section{References.}

[BJ] Bishop, C. J., Jones, P. W., Harmonic measure and arclength. Ann. Math. 132 (1990), 511-547.

[F] Federer, H., Geometric Measure Theory. Springer, 1969.

[G] Gehring, F. W., Lower dimensional absolute continuity properties of quasiconformal mappings. Math. Proc. Camb. Phil. Soc. 78 (1975), 81-93.

[H1] Heinonen, J., Quasiconformal mappings onto John domains. Revista Mat. Iberoamericana 5 (1989), 97-123.

[H2] Heinonen, J., The boundary absolute continuity of quasiconformal map- 
pings. Amer. J. Math. 116 (1994), 1545-1567.

[H3] Heinonen, J., The boundary absolute continuity of quasiconformal mappings II. Revista Mat. Iberoamericana 12 (1996), 697-725.

[HK] Heinonen, J. and Koskela, P., The boundary distortion of a quasiconformal mapping. Pacific J. Math. 165 (1994), 93-114.

[S1] Semmes, S., Bilipschitz mappings and strong $A_{\infty}$-weights. Ann. Acad. Sci. Fenn. Series A I Math. 18 (1993), 211-248.

[S2] Semmes, S., On the nonexistence of bilipschitz parameterizations and geometric problems about $A_{\infty}$-weights. Revista Mat. Iberoamericana 12 (1996), 337-410.

[S3] Semmes, S., Quasisymmetry, measure, and a question of Heinonen. Revista Mat. Iberoamericana 12 (1996), 727-781.

[V] Väisälä, J., Quasi-symmetric embeddings in Euclidean spaces. Trans. Amer. Math. Soc. 264 (1981), 191-204.

Recibido: 20 de noviembre de 1.995

\author{
Juha Heinonen* \\ University of Michigan \\ Department of Mathematics \\ Ann Arbor, MI 48109, U.S.A. \\ juha@math.1sa.umich.edu
}

\footnotetext{
* Supported in part by NSF. The author is a Sloan fellow.
} 